\title{
Percepción de las Prácticas de Gestión Humana y su influencia sobre las Dimensiones Sociales del Clima Organizacional en Empresas Colombianas del Sector Servicios
}

\author{
Lina Marcela Guevara Bedoyal \& Enid Granada Salazar ${ }^{2}$
}

Recibido: 11/01/2017 Aceptado: 05/12/2017

DOI:10.21772/ripo.v36n1a02

\begin{abstract}
Resumen
Se exploró la influencia de las Prácticas de Gestión Humana (PGH) de alto rendimiento sobre las dimensiones sociales del Clima Organizacional (CO), Trato Interpersonal y Apoyo del Jefe, en empresas colombianas del sector servicios. El estudio fue cuantitativo y se usaron regresiones estadísticas. La muestra se conformó por 503 casos pertenecientes a cinco empresas colombianas del sector servicios. Los instrumentos utilizados fueron la Escala de Prácticas de Gestión Humana y la Encuesta de Clima Organizacional (ECO) versión reducida. Prácticas de gestión humana como la vinculación selectiva y la reducción de la brecha de estatus predicen mejor la variable social de Trato Interpersonal. La participación en toma de decisiones y la evaluación de desempeño predicen las percepciones de Apoyo del Jefe. Se concluye que las prácticas de gestión humana inciden de manera importante en el clima organizacional.
\end{abstract}

Palabras clave: prácticas de gestión humana, clima organizacional, apoyo del jefe, trato interpersonal.

\section{Perception of Human Management Practices and their influence on the Social Dimensions of Organizational in Colombian Companies of the Services Sector.}

\begin{abstract}
The influence of high performance human management practices on the social dimensions of the organizational climate, Interpersonal Treatment and Boss Support, in Colombian companies in the service sector was explored. The study was quantitative and statistical regressions were used. The sample consisted of 503 cases belonging to five Colombian companies in the services sector. The instruments used were the Scale of Human Management Practices and THE Organizational Climate Survey (OCS) reduced version. Results showed that human resources management such as selective linkage and reduction of the status gap predict better the social variable of Interpersonal Treatment. Participation in decision-making and performance evaluation predict the Boss Support perceptions. It is concluded that human management practices have a significant impact on the organizational climate..
\end{abstract}

Keywords: human management practices, organizational climate, support of the boss, interpersonal treatment.

La gestión de los recursos humanos no es ajena a la modernización empresarial y a las exigencias del mercado. Cada vez se busca más que esta área se convierta en un aliado estratégico de la organización y tenga repercusiones positivas en otros procesos administrativos y operativos (Calderón, Álvarez, \& Naranjo, 2006; Esch, Wei \& Chiang, 2016). Dado que las prácticas con un enfoque tradicionalista no han logrado dar respuestas eficientes al tipo de metas que las organizaciones se plantean hoy, resulta fundamental que las áreas de gestión humana redefinan sus procesos e innoven en sus prácticas con el fin de obtener resultados óptimos, eficientes, competitivos y una vinculación estrecha con los objetivos organizacionales (Malpica, Rossell \& Hoffmann, 2014; Mayes, Finney, Johnson, Shen, $\& Y i, 2016)$.

Es en este marco que diferentes organizaciones han rediseñado sus procesos enmarcándolos en los sistemas de trabajo de alto desempeño (Ma, Long, Y. Zhang, Zhang \& Lam, 2017).

1 Psicóloga. Especialista en Gerencia del Desarrollo Humano. Universidad EAFIT. Medellín. Colombia. E-mail: lguevara@cincel.com.co

2 Psicóloga, Universidad Católica, Colombia. E-mail: enid.granada@gmail.com

Nota: el presente artículo fue publicado en el 2017. Sin embargo, con el fin de mantener la periodicidad de la Revista, el número corresponde al primer semestre del año 2017

Cómo citar este artículo: Guevara Bedoya, L. \& Granada Salazar, E. (2017). Percepción de las Prácticas de Gestión Humana y su influencia sobre las Dimensiones Sociales del Clima Organizacional en Empresas Colombianas del Sector Servicios. Revista Interamericana de Psicología Ocupacional, 36(1), 22-34, Doi: 10.21772/ripo.v36n1a02 
Desde este contexto, la gestión humana se entiende como un sistema integrado para el manejo del personal que incluye prácticas como reclutamiento y selección, capacitación, formación continua, sistemas de evaluación y participación de los empleados, remuneración, entre otros (Toro, Sanín \& Guevara, 2013). Todo lo anterior, orientado a la mejora de los resultados de los empleados y la empresa a través de la búsqueda de la calidad y el desarrollo del personal, pilares que determinan el plus de estas prácticas y que convierte a los trabajadores en fuente de ventaja competitiva (Crook, Combs, Todd, Woehr \& Ketchen, 2011; Guthrie, 2001).

Las prácticas de gestión humana de alto desempeño están relacionadas con una menor rotación del personal, una mayor productividad y mejores resultados financieros (Chan \& Mak, 2012; Gephart \& Van, 1996; Huselid, 1995). También muestran relaciones con otras variables organizacionales como el aprendizaje (Lorente, Jerez \& Valle, 2005), el compromiso, los comportamientos de ciudadanía organizacional (Fu, 2013), la flexibilidad laboral y el desarrollo cultural (Beltrán, Escrig, Bou \& Roca, 2013)

Las prácticas de gestión humana también han mostrado relación con el clima organizacional (Pons \& Ramos 2012; Takeuchi, Chen, \& Lepak, 2009; Vidaurre, 2009). No obstante, son muy pocas las evidencias empíricas sobre la relación específica entre dichas prácticas y el ambiente de trabajo, por lo que diferentes autores plantean la necesidad de investigación adicional (Calderón et al., 2006; Takeuchi et al., 2009).

Por otra parte, es de importancia identificar que las empresas del sector servicios dentro de Colombia cuentan con un gran potencial de crecimiento debido a que componen el 57,5\% del PIB nacional. Actualmente representan una de las mayores apuestas a nivel comercial, y por su impacto a nivel externo, es importante identificar sus dinámicas internas (Procolombia, 2016). Adicionalmente, este tipo de empresas cuentan con una dinámica particular que hace que sea importante estudiar de forma específica algunos fenómenos psicosociales como los aquí señalados.

El objetivo de esta investigación fue entonces precisar la influencia de las prácticas de gestión humana sobre las dimensiones sociales del clima organizacional en una muestra específica de organizaciones colombianas del sector servicios. Se partió de la hipótesis de que existe una relación positiva entre ambos constructos en el cual las PGH son la causa y el CO el efecto, y que, además, algunas de las variables contempladas dentro de las PGH tienen mayor peso.

\section{Prácticas de Gestión Humana}

Para el presente artículo las prácticas de gestión humana están fundamentadas en los sistemas de alto desempeño (STAD), que se definen como un grupo de prácticas (selección, capacitación, incentivos, remuneración, sistemas de participación, etc.) interrelacionadas con las estrategias organizacionales (Toro et al., 2013) y que tienen como finalidad mejorar el rendimiento de los empleados y la empresa a través de la búsqueda de calidad y el desarrollo del personal (Guthrie, 2001; Crook et al. 2011).

Este enfoque según Gong, Law, Chan y Xin (2009) cuenta con ocho prácticas definidas a continuación, las cuales son las variables base del instrumento de medición Escala de Gestión de Prácticas de Gestión Humana (Toro et al., 2013) utilizado en la presente investigación:

Vinculación selectiva: Existencia de políticas y prácticas administrativas que buscan asegurar una manera objetiva de identificar, evaluar y vincular a los aspirantes más idóneos para cada trabajo.

Participación en toma de decisiones en grupo: Existencia de políticas y prácticas administrativas que promueven y aseguran la participación de todo el personal en las decisiones importantes de sus equipos de trabajo y de la empresa.

Remuneración contingente con el desempeño: Existencia de políticas y prácticas administrativas que vinculan la compensación económica y los beneficios con el desempeño laboral de las personas.

Capacitación extensiva: Existencia de políticas y prácticas de educación que promueven y aseguran la permanencia de altos niveles idoneidad y preparación del personal.

Planeación y avance de carrera: Existencia de políticas y prácticas administrativas que ofrecen claras posibilidades de movilidad y progreso ocupacional del personal dentro de la empresa.

Evaluación del desempeño: Existencia de políticas y prácticas administrativas que valoran objetivamente el desempeño laboral del personal, como requisito para la promoción y la mejora. 
Estabilidad en el empleo: Existencia de políticas

y prácticas administrativas que promueven

$\mathrm{y}$ aseguran altos niveles de permanencia o estabilidad laboral del personal.

Reducción de las diferencias de estatus:

Existencia de políticas y prácticas administrativas que promueven $\mathrm{y}$ aseguran trato igualitario o no discriminatorio entre todo el personal de la organización

Estas prácticas se agrupan en dos categorías: las orientadas a promover el desempeño y las enfocadas en la conservación del personal (Gong et al., 2009). En el primer grupo, se encuentran las variables vinculación selectiva, capacitación extensiva y evaluación de desempeño, y en el segundo participación en toma de decisiones en grupo, estabilidad en el empleo, reducción de la brecha de estatus, planeación y avance de carrera y remuneración contingente con el desempeño (Toro et al., 2013). Vale aclarar que esta agrupación no corresponde a la planteada en el modelo original en el cual la remuneración y el plan de carrera hacían parte de las prácticas orientadas al desempeño. Sin embargo, el modelo escogido fue el que mostró mejores niveles de ajuste en población colombiana (Toro et al., 2013).

\section{Clima Organizacional}

El clima organizacional es una de las condiciones para lograr el éxito empresarial (Toro \& Sanín, 2014). Diversas son las aproximaciones que se encuentran a este constructo, pero convergen en definirlo como un conjunto de percepciones que tienen los colaboradores a partir de los aspectos organizacionales, relaciones grupales y personales (Brunet, 1987; Chiavenato, 2007; Davis, 1991; García 2009; Rousseau, 1988). Tal vez, una de las definiciones más reiteradas en distintos textos académicos es la de Reichers y Schneider (1990), que se refiere al $\mathrm{CO}$ como las percepciones compartidas que construyen los miembros de una organización con respecto a las distintas realidades de su trabajo. Al hablar de un fenómeno cognitivo mediado por la subjetividad de quien interpreta, el $\mathrm{CO}$ puede o no corresponder con la realidad de la organización (Londoño, 2009). En la presente investigación, el $\mathrm{CO}$ se entiende como un conjunto de percepciones que los empleados comparten acerca de diferentes aspectos del trabajo como el liderazgo, las relaciones entre el personal, la retribución, las posibilidades de permanencia, la claridad organizacional, el compromiso, la responsabilidad, los recursos, la coherencia, entre otros fenómenos propios de la vida en el trabajo (Toro \& Sanín, 2014).

El concepto "compartidas" señalado en las definiciones anteriores ha dado lugar a distintas interpretaciones. Las relaciones interpersonales propias de los contextos de trabajo hacen que las personas se comuniquen estas lecturas que hacen del entorno laboral, aun así, ello no garantiza que exista consenso alrededor de dichas percepciones. La variabilidad en muchas de las organizaciones es alta, y eso es parte de lo que debe reconocerse para hablar de CO. Existen entonces modelos de agregación y modelos de composición, y cada uno hace una interpretación distinta del concepto de "compartidas". Mientras que para los primeros se refiere a consenso, para los segundos se refiere más a la interacción social que hace que estás interpretaciones se comuniquen (González-Romá, 2011).

Desde algunas posturas teóricas como la planteada es necesario cierto nivel de acuerdo para poder hablar de clima organizacional, sin embargo, desde otras perspectivas se asume que, aún en el disenso, el que el objeto de la interpretación sean las realidades organizaciones y no la condición individual, permite hablar de clima organizacional en lugar de clima psicológico. Así, en esta conceptualización al hablar de clima psicológico o de clima organizacional lo que cambia es el referente por el que se pregunta independiente del nivel de heterogeneidad en las percepciones. Para los primeros casos donde hay homogeneidad se puede hablar de composición, mientras que para los segundos donde prima la heterogeneidad se puede hablar de compilación (Kozlowski \& Klein, 2000).

Diversas investigaciones aseguran que el $\mathrm{CO}$ está relacionado con la satisfacción, el rendimiento (Brunet, 1987), la motivación laboral (Chiavenato, 2007; Toro \& Sanín, 2014).con una mejor calidad de servicio (Salanova \& Peiró, 2005), la imagen positiva de la organización y un mejor posicionamiento de la empresa (Toro \& Sanín, 2014). Sin embargo, pese a todos los efectos documentados del CO, poca información se tiene respecto de las prácticas de gestión humana que le anteceden. Algunos estudios enuncian la interacción entre las PGH y el CO pero no se especifican dichas relaciones (Pons \& Ramos, 2012; Sepúlveda, 2015; Takeuchi et al., 2009).

Para efectos de esta investigación se trabajó con un modelo que contempla diez variables de clima organizacional (trato interpersonal, apoyo del jefe, sentido de pertenencia, retribución, disponibilidad de recursos, estabilidad, coherencia, 
claridad organizacional, trabajo en equipo y valores colectivos). No obstante, los análisis se llevaron a cabo con las variables sociales: Trato Interpersonal y con Apoyo del Jefe, por su alta capacidad de incidir sobre la calidad global del clima y su contribución a explicar las percepciones de compromiso del personal (Toro \& Sanín, 2014).

Trato Interpersonal es entendida como la "percepción del grado en que el personal se ayuda entre sí y sus relaciones son de cooperación y respeto" (Toro, 2008). Dos aspectos clave se destacan en esta definición: la cooperación y el respeto que se tienen dentro del grupo de trabajo. La cooperación hace referencia a la colaboración, apoyo y ayuda mutua y constante que tiene un equipo de trabajo. El respeto es la consideración y amabilidad que se gestan en las relaciones interpersonales (Toro \& Sanín, 2014).

Apoyo del Jefe es definida como la "percepción del grado en que el jefe respalda, estimula y da participación a sus colaboradores" (Toro, 2008). Tres son las dimensiones que envuelve esta variable y que hace parte de ese liderazgo que caracteriza un jefe: apoyo, estímulo y posibilidades de participación. La primera hace referencia a las percepciones que los colabores tienen acerca de la orientación, acompañamiento, y respaldo que brinda el jefe, por otra parte el estímulo son aquellas percepciones que los colabores tienen frente al reconocimiento brindado por el jefe a la hora de realizar labores, y finalmente las posibilidades de participación son entendidas como esas percepciones que se tiene sobre la disponibilidad que tiene el jefe para escuchar las ideas u opiniones de los colaboradores (Toro \& Sanín, 2014).

Las percepciones que se tengan del apoyo y gestión del jefe repercuten en el desempeño, lealtad, compromiso, y satisfacción del personal (Goleman, 2007; Toro \&Sanín 2014) por tal motivo la importancia de generar que los jefes desarrollen habilidades de apoyo, sociales, comunicativas y que generen estrategias de participación en los colaboradores. (Toro \& Sanín, 2014).

El objetivo de esta investigación es entonces precisar las relaciones existentes entre las prácticas de gestión humana y las variables sociales del clima organizacional en una muestra específica de organizaciones colombianas del sector servicios. Se parte de la hipótesis de que existe una relación positiva entre ambos constructos y que, además, algunas de las variables contempladas dentro de las PGH tienen mayor efecto sobre el CO.

\section{Método}

El estudio tuvo un enfoque cuantitativo, transversal, no experimental, (Hernández, Fernández \& Baptista, 2014).

\section{Participantes}

Se contó con una muestra de 503 casos pertenecientes a cinco empresas colombianas del sector servicios. El $45 \%$ de los participantes pertenecían a entidades privadas, el 33\% a entidades públicas, y el $21 \%$ a empresas mixtas. El $90 \%$ de los participantes tenían contrato a término indefinido, el $9.7 \%$ a término fijo. El $47 \%$ tenían estudios de posgrado, el $23 \%$ una formación técnica o tecnológica, el $18 \%$ pregrado y un $9 \%$ secundaria. El $57 \%$ de los participantes reportaron tener personas a cargo. El $38 \%$ llevaban entre 6 a 10 años de antigüedad, el 28\% entre 21 y más años y el 33\% entre 11 y 20 años.

\section{Procedimiento}

La recolección de información fue realizada por Cincel S.A.S. (Centro de Investigación en Comportamiento Organizacional), quienes realizaron una investigación más amplia tomando 3134 casos pertenecientes a 23 organizaciones del sector educativo, servicios e industrial de países como Chile, Colombia, México y República Dominicana.

Para el acceso a las organizaciones, Cincel realizó un muestreo por disponibilidad a través de una invitación de participación a la gerencia de cada organización y para la selección de los participantes se realizó un muestreo al azar, disminuyendo el sesgo, y buscando obtener un número de participantes proporcional en cada organización. La aplicación de los instrumentos fue realizada al $35 \%$ de la muestra de forma virtual y al resto de la población de forma presencial con el apoyo de un equipo de profesionales expertos en aplicación de encuestas. Los instrumentos empleados fueron previamente validados para ambas modalidades de administración. Antes de la aplicación se explicó a los participantes el propósito de la investigación y los derechos que tenían, pidiéndoles su debido consentimiento. La lectura de hojas de respuestas físicas y la consolidación de los datos virtuales estuvo también a cargo del área Logística de Cincel S.A.S., quienes usaron los programas Survey Monkey, Teleform y SPSS v. 22.

Para efectos del presente estudio se realizó una propuesta de investigación que fue enviada a Cincel S.A.S., junto a una petición formal de vinculación 
al proyecto solicitando acceso a los datos con el compromiso de producir un artículo científico a partir del análisis de los mismos.

Es importante mencionar que tanto el proyecto global como la investigación, fueron revisados y avalados por el comité científico de Cincel, quienes verificaron que cumplieran con los lineamientos éticos y con lo dispuesto en la ley 1090 del 2006, que rige el ejercicio de la psicología en Colombia.

\section{Instrumentos}

Para la variable de prácticas de gestión humana se hizo uso del instrumento Escala de prácticas de gestión humana, la cual consta de 48 reactivos entre los que se encuentran afirmaciones como "En esta empresa hay un proceso de selección estricto para vincular al personal", a las que se responde en un formato tipo Likert y que cuenta con cinco opciones de respuesta (0) ni de acuerdo, ni en desacuerdo, (1) completamente en desacuerdo, (2) en desacuerdo, (3) de acuerdo y (4) completamente de acuerdo. Esta escala busca medir ocho variables: estabilidad en el empleo, reducción de las diferencias de status, vinculación selectiva, participación en toma de decisiones en grupo, remuneración contingente con el desempeño, capacitación extensiva, planeación y avance de carrera y evaluación de desempeño. Fue validada en población colombiana con 1,873 casos pertenecientes a 11 empresas de este país, y mostró con un coeficiente interno de consistencia de 0.96 a través del método Alfa de Cronbach. La validez se verificó con análisis factorial exploratorio a través del método de extracción de Ejes Principales y rotación Promax el cual mostró una adecuada configuración factorial (Toro et al. 2013).

Para la medición de clima organizacional se hizo uso de la encuesta ECO versión reducida, la cual fue validada en una muestra de 44,244 casos, que corresponden a 94 empresas colombianas y otros países latinos, contando con un Alfa de Cronbach de 0,93 , una adecuada configuración factorial y una validación a través del método de extracción de ejes principales con rotación Oblimín Directo. Esta cuenta afirmaciones como "En mi trabajo hay cooperación entre el personal" que se responden con una escala tipo Likert de 4 puntos, donde 1 es completamente en desacuerdo y 4 completamente de acuerdo. Cuenta con 36 reactivos y mide variables como trato interpersonal, apoyo del jefe, sentido de pertenecía, retribución, disponibilidad de recursos, estabilidad, coherencia, claridad organizacional, trabajo en equipo (López, 2004; CINCEL S.A.S, 2014). Sin embargo, para los análisis se tomaron solamente los ítems de Apoyo del Jefe y Trato Interpersonal.

\section{Análisis de información}

Se calculó el coeficiente de correlación intraclase (CCI) para verificar que la muestra proveniente de las distintas empresas del sector servicios realmente permitía la agregación de los datos. Dado que era la misma muestra para todas las variables y que no se tuvo acceso al total de la población, se calculó el CCI2 (Landers 2015). Inicialmente se calcularon las correlaciones entre las variables y posteriormente se utilizó una regresión por pasos sucesivos buscando cuáles de las variables de PGH tenían mayor capacidad de predicción de las dos variables de CO. Esta forma de la regresión lineal múltiple permite generar diversos moldeos cuyas medidas de ajuste determinan cuáles de los predictores introducidos tienen mayor capacidad de explicar la variabilidad de la dependiente. Además, genera medidas de redundancia entre las variables del modelo a fin de que sea posible seleccionar la opción más parsimoniosa.

\section{Resultados}

El coeficiente de correlación intraclase indicó que para todas las variables era posible agrupar el compendio de empresas como una sola muestra. La Tabla 1 muestra los resultados para cada una de las dimensiones exploradas. Todos los valores para el CCI2 se consideran favorables de acuerdo con los criterios reportados en la literatura (James, Demaree \& Wolf, 1984).

\section{Tabla 1}

Índices de correlación intraclase para las variables del estudio

\begin{tabular}{ll}
\hline Variable & CCI2 \\
\hline 1 Apoyo del Jefe & 0.777 \\
2 Trato Interpersonal & 0.697 \\
3. PGH Estabilidad en el empleo & 0.966 \\
4 PGH Reducción de la brecha de estatus & 0.700 \\
5 PGH Vinculación selectiva & 0.874 \\
6 PGH Toma de decisiones & 0.714 \\
7 PGH Remuneración contingente con el & 0.881 \\
desempeño & 0.920 \\
8 PGH Capacitación extensiva & 0.687 \\
9 PGH Plan de carrera & 0.908 \\
10 PGH Evaluación de desempeño &
\end{tabular}


Tabla 2

Correlaciones entre las variables del estudio, muestra (N), medias (M) y desviaciones estándar (DE)

\begin{tabular}{|c|c|c|c|c|c|c|c|c|c|c|c|c|}
\hline & $\mathrm{N}$ & $\mathrm{M}$ & $\mathrm{DE}$ & 1 & 2 & 3 & 4 & 5 & 6 & 7 & 8 & 9 \\
\hline 1 Apoyo del Jefe & 503 & 2.86 & .70 & - & & & & & & & & \\
\hline $\begin{array}{l}2 \text { Trato } \\
\text { Interpersonal }\end{array}$ & 502 & 3.34 & .57 & $.584 * *$ & - & & & & & & & \\
\hline $\begin{array}{l}3 \text { PGH Estabilidad } \\
\text { en el empleo }\end{array}$ & 503 & 3.09 & .63 & $.334 * *$ & $.400 * *$ & - & & & & & & \\
\hline $\begin{array}{l}4 \text { PGH Reducción } \\
\text { de la brecha de } \\
\text { estatus }\end{array}$ & 503 & 2.86 & .55 & $.499 * *$ & $.452 * *$ & $.554 * *$ & - & & & & & \\
\hline $\begin{array}{l}5 \text { PGH Vinculación } \\
\text { selectiva }\end{array}$ & 502 & 3.01 & .63 & $.515^{* *}$ & $.477 * *$ & $.549 * *$ & $.568 * *$ & - & & & & \\
\hline $\begin{array}{l}6 \text { PGH Toma de } \\
\text { decisiones }\end{array}$ & 502 & 2.54 & .58 & $.547 * *$ & $.437 * *$ & $.510 * *$ & $.659 * *$ & $.536^{* *}$ & - & & & \\
\hline $\begin{array}{l}7 \text { PGH } \\
\text { Remuneración } \\
\text { contingente con el } \\
\text { desempeño }\end{array}$ & 501 & 2.3634 & .67 & $.423 * *$ & $.297 * *$ & $.446^{* *}$ & $.566^{* *}$ & $.486^{* *}$ & $.602 * *$ & - & & \\
\hline $\begin{array}{l}8 \text { PGH } \\
\text { Capacitación } \\
\text { extensiva }\end{array}$ & 502 & 2.96 & .55 & $.429 * *$ & $.371 * *$ & $.578 * *$ & $.554 * *$ & $.576 * *$ & $.578 * *$ & $.577 * *$ & - & \\
\hline $\begin{array}{l}9 \text { PGH Plan de } \\
\text { carrera }\end{array}$ & 500 & 2.47 & .68 & $.542 * *$ & $.451 * *$ & $.523 * *$ & $.688^{* *}$ & $.671 * *$ & $.702 * *$ & $.619 * *$ & $.653 * *$ & - \\
\hline $\begin{array}{l}10 \text { PGH } \\
\text { Evaluación de } \\
\text { desempeño }\end{array}$ & 499 & 2.74 & .68 & $.554 * *$ & $.414^{* *}$ & $.482 * *$ & $.630 * *$ & $.668 * *$ & $.670 * *$ & $.639 * *$ & $.612 * *$ & $.702 * *$ \\
\hline
\end{tabular}

Nota: Correlaciones: ${ }^{* *} \mathrm{p}<0.01$.

Todas las variables del presente estudio Plany AvancedeCarrera, y Evaluación de Desempeño. mostraron correlaciones entre sí $(p<0,01)$. Las Las de menor correlación corresponden a Apoyo del variables con mayor correlación son Jefe y Estabilidad en el empleo.

Tabla 3

Regresión por pasos sucesivos de las variables de PGH sobre la variable Trato Interpersonal

\begin{tabular}{|c|c|c|c|c|c|c|c|c|c|c|c|c|c|}
\hline Modelo & & $\mathbf{R}$ & $\mathbf{R}^{2}$ & $\mathbf{E}$ & M2 & $\mathbf{F}$ & Sig. & B & t & $\begin{array}{l}\text { Sig. } \\
\text { (B) }\end{array}$ & $\begin{array}{l}\text { Durbin- } \\
\text { Watson }\end{array}$ & Tol. & VIF \\
\hline 1 & VS & $.479^{a}$ & .230 & .49734 & 36.258 & 146.585 & $.000^{\mathrm{b}}$ & .479 & 12.107 & .000 & & 1.000 & 1.000 \\
\hline \multirow[t]{2}{*}{2} & VS & $.530^{\mathrm{b}}$ & .281 & .48121 & 22.121 & 95.528 & $.000^{\mathrm{c}}$ & .322 & 6.869 & .000 & & .670 & 1.493 \\
\hline & ES & & & & & & & .275 & 5.872 & .000 & & .670 & 1.493 \\
\hline \multirow[t]{3}{*}{3} & VS & $.543^{\mathrm{c}}$ & .295 & .47695 & 15.489 & 68.088 & $.000^{\mathrm{d}}$ & .282 & 5.860 & .000 & & .623 & 1.604 \\
\hline & ES & & & & & & & .190 & 3.535 & .000 & & .499 & 2.003 \\
\hline & TD & & & & & & & .164 & 3.128 & .000 & 2.108 & .527 & 1.896 \\
\hline
\end{tabular}

Nota $: \mathrm{R}=$ Valor de la correlación, R2= Valor de la regresión, $\mathrm{E}=$ Error estándar, $\mathrm{M} 2=$ media cuadrática, $\mathrm{F}=\mathrm{Estadístico} \mathrm{de} \mathrm{prueba,} \mathrm{Sig=} \mathrm{Significancia,} \mathrm{B}=\mathrm{Beta}, \mathrm{t}=$ Estadístico de prueba, Sig (B)= Significancia del Beta. Tol= Tolerancia. VIF= Indicador de colinealidad, VS=Vinculación selectiva, ES= Reducción de la brecha de estatus, $\mathrm{TD}=$ Toma de decisiones. 
En la tabla 3, se pueden observar los tres modelos generados que integran las variables vinculación selectiva, reducción de la brecha de estatus y toma de decisiones. Aunque todos son significativos a nivel estadístico, se encuentran datos que generan una inclinación sobre el modelo 2. Vinculación selectiva y reducción de la brecha de estatus explican en un $28,1 \%$ de los cambios en la variable dependiente (Trato interpersonal) en comparación al modelo 1 donde hay una menor explicación de la varianza $\left(\mathrm{R}^{2}=23.0\right)$. Por otra parte en el modelo 3, aunque la varianza explicada es mayor (29.5\%), su indicador de colinealidad $(\mathrm{VIF}=2.003)$
Evidentemente cada vez que se da un paso en este tipo de análisis y se incluye una nueva variable predictora dentro del modelo, se reduce la capacidad explicativa de la variable anterior, pero a cambio se incrementa la del modelo. De ahí, la importancia de revisar algunas medidas de ajuste para tomar la decisión estadística más adecuada. Se escoge así un modelo con suficiente capacidad explicativa pero más parsimonioso.

El beta indica que la variable con mayor capacidad de predicción en el modelo escogido es la vinculación selectiva $(B=.322)$ seguida de la reducción de la brecha de estatus $(\mathrm{B}=.275)$.

\section{Tabla 4}

Regresión por pasos sucesivos de las variables de PGH sobre la variable Apoyo del Jefe

\begin{tabular}{|c|c|c|c|c|c|c|c|c|c|c|c|c|c|}
\hline Modelo & & $\mathrm{R}$ & $\mathrm{R}^{2}$ & E & M2 & F & Sig. & B & $\mathrm{t}$ & Sig. (B) & $\begin{array}{l}\text { Durbin- } \\
\text { Watson }\end{array}$ & Tol. & VIF \\
\hline 1 & ED & $.558^{\mathrm{a}}$ & .312 & .59142 & 77.952 & 222.857 & $.000^{\mathrm{b}}$ & .558 & 14.928 & .000 & & 1.000 & 1.000 \\
\hline \multirow[t]{2}{*}{2} & ED & $.605^{\mathrm{b}}$ & .366 & .56829 & 45.739 & 141.628 & $.000^{\mathrm{c}}$ & .348 & 7.178 & .000 & & .550 & 1.819 \\
\hline & $\mathrm{TD}$ & & & & & & & .314 & 6.472 & .000 & & .550 & 1.819 \\
\hline \multirow[t]{3}{*}{3} & ED & $.626^{\mathrm{c}}$ & .391 & .55732 & 32.616 & 105.010 & $.000^{\mathrm{d}}$ & .228 & 4.186 & .000 & & .419 & 2.384 \\
\hline & $\mathrm{TD}$ & & & & & & & .277 & 5.755 & .000 & & .535 & 1.870 \\
\hline & VS & & & & & & & .217 & 4.529 & .000 & & .542 & 1.847 \\
\hline \multirow[t]{4}{*}{4} & ED & $.630^{\mathrm{d}}$ & .397 & .55548 & 24.789 & 80.338 & $.000^{\mathrm{e}}$ & .204 & 3.676 & .000 & & .401 & 2.494 \\
\hline & $\mathrm{TD}$ & & & & & & & .236 & 4.540 & .000 & & .456 & 2.194 \\
\hline & VS & & & & & & & .195 & 3.990 & .000 & & .516 & 1.938 \\
\hline & ES & & & & & & & .105 & 2.059 & .000 & 2,122 & .478 & 2.094 \\
\hline
\end{tabular}

Nota $: \mathrm{R}=$ Valor de la correlación, $\mathrm{R} 2=$ Valor de la regresión, $\mathrm{E}=$ Error estándar, $\mathrm{M} 2=$ media cuadrática, $\mathrm{F}=\mathrm{Estadístico} \mathrm{de} \mathrm{prueba,} \mathrm{Sig=} \mathrm{Significancia,} \mathrm{B}=\mathrm{B}$ eta, $\mathrm{t}=\mathrm{Es}-$ tadístico de prueba, Sig $(B)=$ Significancia del Beta. Tol= Tolerancia, VIF= Indicador de colinealidad, ED= Evaluación de desempeño, TD= Toma de decisiones, VS= vinculación selectiva, $\mathrm{ES}=$ Reducción de la brecha de estatus.

Respecto de Apoyo del Jefe, el modelo 1 explica la varianza de la variable dependiente en un $31 \%$, el modelo 2 en un $37 \%$, el modelo 3 en un $39 \%$ y el 4 en un $40 \%$. Sin embargo, se determina que el modelo 2 es el más adecuado dado que si se compara con el modelo $3\left(\mathrm{R}^{2}=0.39-\mathrm{VIF}=2.384\right)$ y el modelo $4\left(\mathrm{R}^{2}=0.40-\mathrm{VIF}=2.94\right)$, en estos dos el $\mathrm{R}^{2}$ no tiene un aumento significativo y su indicador de colinealidad permite evidenciar una redundancia entre las variables independientes.

Finalmente en este análisis la variable con mayor capacidad de predicción es evaluación de desempeño $(\mathrm{B}=.348)$ seguido por toma de decisiones $(\mathrm{B}=.314)$.

\section{Discusión}

El presente estudio tenía como propósito encontrar la influencia de las prácticas de gestión humana sobre las dimensiones sociales de clima organizacional, apoyo del jefe y trato interpersonal, identificando además aquellas que poseían mayor capacidad de predicción, en una muestra de empresas colombianas del sector servicios. Los datos obtenidos permitieron confirmar que existe tal influencia, y señalaron que vinculación selectiva y reducción de las brechas de estatus tienen mayor incidencia sobre la variable Trato Interpersonal, y evaluación de desempeño y toma de decisiones sobre Apoyo del Jefe. 
es alto lo que señala una posible redundancia entre las variables independientes.

Aunque anteriormente otros estudios mencionados ya habían señalado la relación entre las prácticas de gestión humana y el clima organizacional (Pons \& Ramos, 2012; Sepúlveda, 2015; Takeuchi et al., 2009), este estudio permitió profundizar un poco más en dicha relación, pues se hace un análisis particular de las empresas del sector servicios detallando las dimensiones con mayor capacidad predictiva.

La existencia de prácticas que permitan identificar, evaluar y vincular aspirantes idóneos para el cargo (vinculación selectiva) y la promoción de un trato igualitario en toda la organización (reducción de la brecha de estatus), generan una influencia en la percepción que los empleados tienen sobre el trato interpersonal. De igual manera, el contar con políticas que promuevan y aseguren la participación de todo el personal (toma de decisiones) y la valoración objetiva del desempeño laboral (evaluación de desempeño) genera percepciones positivas sobre el apoyo brindado por los jefes.

Lo encontrado con la variable reducción de la brecha de estatus guarda relación con otros estudios que abordan el tema de las distancias de poder, pues, aunque no lo relacionan de forma directa con el clima organizacional, está documentado que culturas en las que se percibe menor distancia se caracterizan también por relaciones más cercanas y favorables entre los miembros de la organización (Espinosa, Contreras, \& Barbosa, 2015). Las acciones encaminadas a reducir el trato diferencial entre las personas según la jerarquía, favorecen un trato más respetuoso y cooperativo pues se genera cercanía y se facilita la interacción y el relacionamiento.

En cuanto a la variable vinculación selectiva, se plantea que el contar con un proceso que permita vincular candidatos potencialmente calificados para un cargo, favorece el mantenimiento de un adecuado clima de trabajo en tanto la congruencia entre el perfil de la personal y del cargo facilita la adaptación a la cultura (Fernández, 2010). Integrar una nueva persona al equipo no solo impacta los resultados laborales del grupo, sino que genera efectos importantes sobre las formas de relacionarse en el mismo. De allí que cuando las personas perciben que la decisión sobre la vinculación de un nuevo integrante se realiza con criterios objetivos, su acogida e integración se de en términos más favorables y se propicie la cooperación y el respeto como parte de la dinámica del grupo.
Por su parte, la existencia de políticas y prácticas que permiten valorar objetivamente el desempeño laboral del personal ha mostrado diferentes beneficios, entre ellos un impacto positivo en el clima organizacional (Chiavenato, 2007), relación que también fue hallada en el presente estudio. Una adecuada estrategia de evaluación permite mejorar el desempeño del personal y del área en general, lo que implica el mejoramiento, no solo del desempeño de tarea sino también social (Toro, 1990). Impacta también la promoción, ascensos o reubicaciones que a su vez inciden en la motivación hacia el trabajo y el compromiso con la labor (Bohlander \& Sherman, 2001; Chiavenato, 2007). A lo anterior, se añade que este proceso tiene una relación importante con la percepción sobre el apoyo del jefe, lo cual podría derivarse del hecho de que son precisamente los jefes los encargados de desarrollar la evaluación aun cuando los lineamientos o la estrategia haya sido definida desde gestión humana o desde otras instancias organizacionales.

Lo anterior sucede también con los procesos de participación en toma de decisiones. Los hallazgos de este estudio guardan relación con investigaciones como las de Litwin y Stringer (1978) quienes señalan que la variable mencionada tiene relación con el clima organizacional, dado que al promover políticas y oportunidades de participación se genera en los empleados un comportamiento participativo y activo, un aumento en la satisfacción laboral y una mejor percepción de la organización en tanto que se sienten integrados a los procesos de esta (Quiroa, 2014). Sin embargo, quienes finalmente permiten que se materialice o se obstaculicen las practicas participativas son los jefes de cada equipo. De allí que la percepción sobre el apoyo del jefe se vea favorablemente impactada cuando las personas perciben que en la organización cuentan con posibilidades reales de participación e incidencia en los procesos de toma de decisiones.

Si bien, los hallazgos son interesantes, el estudio cuenta con algunas limitaciones. Todos los datos provienen de encuestas de auto-informe y de una muestra colombiana. Aunque los instrumentos empleados cuentan con adecuadas propiedades psicométricas, sería enriquecedor replicar el estudio utilizando otras fuentes de información. Se sugiere también contemplar otras variables como antigüedad, tipo de contrato entre otras, que pueden ayudar a comprender aún más los fenómenos estudiados.

Otra limitación del estudio consiste en que para los análisis aquí efectuados no se hicieron cálculos y análisis de otros conceptos importantes 
asociadas a la compilación de datos en CO (medidas de acuerdo intra-unidad o específicamente de la fuerza del clima).

Aunque en las entregas individuales previamente hechas a cada una de las empresas participantes del estudio se tuvieron en cuenta estas medidas para enriquecer los análisis y orientar las intervenciones en dichas entidades, para efectos de los análisis de regresión estadística aquí empleados se trabajó exclusivamente con las medias de los grupos, previa verificación del índice de correlación intraclase entre las cinco organizaciones participantes.

\section{Conclusiones}

El sistema organizacional y específicamente las prácticas de gestión humana, repercuten en las percepciones que los empleados se forman acerca de la organización. Generar unas prácticas equitativas cuyo foco esté en el desarrollo del personal puede favorecer la formación de percepciones positivas por parte del colaborador. Dentro del presente estudio las prácticas de gestión humana como la vinculación selectiva y reducción de la brecha estatus son predictores de la variable trato interpersonal. Por su parte, toma de decisiones y evaluación de desempeño predicen la variable apoyo del jefe.

Las prácticas de gestión humana se realizan de forma mecánica en muchas organizaciones sin mayores claridades de los efectos que tienen sobre algunos fenómenos psicosociales. Conocer que los efectos de lo que se hace como parte de la gestión humana permite enfocar mejor los procesos a fin de incidir en el ambiente de trabajo y por tanto en la calidad de vida de las personas y en la productividad de las empresas. Igualmente, gestionar el clima organizacional, requiere también no solo de una participación activa de la gente sino de una verificación previa del estado de las prácticas de gestión humana pues estas suelen ser el soporte para cualquier intervención de orden psicosocial en la organización.

Estudios como este tienen importantes implicaciones prácticas pues permiten a las empresas seleccionar con mayor criterio aquellos procesos en los que invertirán mayor o menor esfuerzo cuando sus focos de intervención se centran en los aspectos sociales del CO.

\section{Referencias}

Beltrán, I., Escrig, A., Bou, J., \& Roca, V. (2013). Influencia de las prácticas de recursos humanos en la flexibilidad de los empleados.
Cuadernos de Economía y Dirección de la Empresa, 16(4), 221- 237.

Bohlander, G., \& Sherman, A. (2001). Administración de los Recursos Humanos (3a ed.). México: Prentice-Hall Hispanoamericana.

Brunet, L. (1987). El clima de trabajo en las organizaciones: Definición, Diagnóstico y Consecuencias. México: Editorial Trillas.

Calderón,G.,Álvarez,C., \& Naranjo,J.(2006). Gestión humana en las organizaciones un fenómeno complejo: evolución, retos, tendencias y perspectivas de investigación. Cuadernos de Administración, 19(32), 225 - 254.

Chan, S. C., \& Mak, W. M. (2012). High performance human resource practices and organizational performance: The mediating role of occupational safety and health. Journal of Chinese Human Resources Management, 3(2), 136-150. Doi: 10.1108/20408001211279238

Chiavenato, I. (2007). Administración de recursos humanos (8a ed.). México: McGraw-Hill.

CINCELS.A.S. (2014). Ficha Técnica del instrumento Escala ECO. Documento inédito.

Crook, R., Combs, J., Todd, S., Woehr, D., \& Ketchen, J. (2011). Does Human Capital Matter? A Meta-Analysis of the Relationship between Human Capital and Firm Performance. Journal of Applied Psychology, 96(3), 443456.

Davis, K. (1991). Comportamiento Humano en el Trabajo. México: Mc Graw Hill.

Esch, E., Wei, L., \& Chiang, F. (2016). Highperformance human resource practices and firm performance: the mediating role of employees' competencies and the moderating role of climate for creativity. The International Journal of Human Resource Management, 1-26. Doi: 0.1080/09585192.2016.1206031

Espinosa, J. C., Contreras, F., \& Barbosa, D. (2015). Prácticas de liderazgo y su relación con la cultura en un grupo de países latinoamericanos. Diversitas: Perspectivas en Psicología, 11(2), 303-317. Doi:10.15332/ s1794-9998.2015.0002.10

Fernández, J. (2010). Reclutamiento y selección de personal. Madrid: Pirámide. 
Fu, Y-K. (2013). High-performance human resource practices moderate flight attendants' organizational commitment and organizational citizenship behavior. Social Behavior and Personality: an international journal, 41(7), 1195-1208.

García, M. (2009). Clima Organizacional y su Diagnóstico: Una aproximación Conceptual. Cuadernos de Administración, 42, 43-6.1

Gephart, M., \& Van, M. (1996). The power of high performance work system. Training \& Development, 50(10), 21-36.

Goleman, D. (2007). La inteligencia emocional en la empresa. Buenos Aires: Zeta.

Gong, Y., Law, K., Chang, S., \& Xin, K. (2009). Human resources management and firm performance: the differential role of managerial affective and continuance commitment. Journal of Applied Psychology, 94(1), 263-275.

González-Romá, V. (2011). El clima de los equipos de trabajo: una propiedad configuracional. Papeles del Psicólogo, 32(1), 48-58.

Guthrie, J. (2001). High-involvement work practices, turnover, and productivity: Evidence from New Zealand. Academy of Management Journal, 44(1), 180-190.

Hernández, R., Fernández, C., \& Baptista, P. (2015). Metodología de la investigación (5a ed.). Mexico: Mcgraw-Hill.

Huselid, M. (1995). The impact of human resource management practices on turnover, productivity, and corporate financial performance. Academy of Management Journal, 38(3), 635-872.

James, L. R., Demaree, R. G., \& Wolf, G. (1984). Estimating within-group interrater reliability with and without response bias. Journal of Applied Psychology, 69(1), 85-98.

Kozlowski, S. W. J., \& Klein, K. J. (Eds.) (2000). A multilevel approach to theory and research in organizations. Contextual, temporal, and emergent processes. In Multilevel theory, research, and methods in organizations (pp. 3-90). San Francisco: Jossey-Bass.

Landers, R. (2015). Computing intraclass correlations (ICC) as estimates of interrater reliability in SPSS. The Winnower. Doi: 10.15200/ winn. 143518.81744

Litwin, G., \& Stringer, R. (1978). Organizational climate. New York: Simon \& Schuster.

Londoño, M. (2009) Relaciones entre clima organizacional, acoso psicológico y los Comportamientos éticos en el trabajo. Estudio de un caso. Revista interamericana de psicología ocupacional, 28(2), 198-212.

López, R. (2004) Actualización psicométrica de la encuesta ECO de clima organizacional. Revista Interamericana de Psicología Ocupacional, 23(2), 94-100.

Lorente, C., Jerez, G., \& Valle, C. (2005). Las prácticas de Recursos Humanos de Alto Rendimiento y la capacidad de aprendizaje organizativo: incidencia e implicaciones. Cuadernos de Economía y Dirección de la Empresa, 24(24), 29-55.

Ma, Z., Long, L., Zhang, Y., Zhang, J., \& Lam, C. K. (2017). Why do high-performance human resource practices matter for team creativity? The mediating role of collective efficacy and knowledge sharing. Asia Pacific Journal of Management, 34(3), 565-586. Doi: 10.1007/ s10490-017-9508-1.

Malpica, R., Rossell, R., \& Hoffmann, I. (2014). Equipos de trabajo de alto desempeño. Observatorio Laboral Revista Venezolana, 7(14), 69-83.

Mayes, B. T., Finney, T. G., Johnson, T. W., Shen, J., \& Yi, L. (2016). The effect of human resource practices on perceived organizational support in the People's Republic of China. The International Journal of Human Resource Management, 28(9), 1-30. Doi: 10.1080/09585192.2015.1114768

Pons, F. \& Ramos, J. (2012). Influencia de los Estilos de Liderazgo y las Prácticas de Gestión de RRHH sobre el Clima Organizacional de Innovación. Revista de psicología del Trabajo $y$ de las Organizaciones, 28(2), 81-98.

Procolombia (2016). Inversión en el sector Servicios en Colombia. Recuperado de http://www. inviertaencolombia.com.co/sectores/ servicios.html

Quiroa, C. (2014). Toma de decisiones y productividad laboral (Tesis, Universidad Rafael Landívar). Recuperado de http://biblio3.url.edu.gt/ 
Tesario/2014/05/43/Quiroa-Claudia.pdf

Reichers, A., \& Schneider, B. (Eds.). (1990). Climate and culture: An evolution of constructs. In Organizational climate and culture (pp. 5-39). San Francisco: Jossey Bass.

Rousseau, D. (1988). The construction of climate in organizational research. In C. L. Cooper y I. Robertson (Eds.), International Review of Industrial and Organizational Psychology (pp. 137-158). England: John Wiley \& Sons.

Salanova, M., \& Peiró, J. (2005). Linking organizational resources and work engagement to employee performance and customer loyalty: the mediation of service climate. Journal of Applied Psychology, 90(6), 12171227.

Sepúlveda, G. (2015). Análisis de la influencia de las prácticas de gestión humana en el desempeño en 3 organizaciones chilenas. (Tesis de maestría, Universidad Alberto Hurtado, Chile). Recuperado de http://repositorio. uahurtado.cl/handle/11242/6848

Takeuchi, R., Chen, G. \& Lepak, D. (2009). Through the looking glass of a social system: cross-level effects of high-performance work systems on employees' attitudes. Personnel Psychology, 62, 1-29.

Toro, F. (1990). Desempeño y Productividad. Medellín-Colombia: Cincel.

Toro, F. (2008). Análisis Psicométrico de la Encuesta ECO IV de Clima Organizacional por países. Revista Interamericana de Psicología Ocupacional, 27(1), 44-57.

Toro, F., \& Sanín, A. (2014). Gestión del clima organizacional intervención basada en evidencias. Colombia: Cincel.

Toro, F., Sanín, A., \& Guevara, L. (2013). Validación de una Escala Para Evaluación de la Existencia, Calidad y Cobertura de las Prácticas de Gestión Humana en las Organizaciones. Revista interamericana de psicología ocupacional, 32(1), 9-30.

Vidaurre, R. (2009). Diagnóstico del Clima Organizacional en una Empresa de Telecomunicaciones de el Salvador (Tesis de maestría, Universidad Dr. Jose Matias Delgado). Recuperado de http://webquery.
ujmd.edu.sv/siab/bvirtual/BIBLIOTECA $\% 20$ VIRTUAL/TESIS/03/MPO/ADVD0001155. pdf 